\title{
(6) OPEN ACCESS \\ Randomised trial of cord clamping and initial stabilisation at very preterm birth
}

\author{
Lelia Duley, ${ }_{1}^{1}$ Jon Dorling, ${ }^{2}$ Angela Pushpa-Rajah, ${ }^{3}$ Sam J Oddie, ${ }^{4}$ \\ Charles William Yoxall, ${ }^{5}$ Bernard Schoonakker, ${ }^{6}$ Lucy Bradshaw, ${ }^{1}$ Eleanor J Mitchell, ${ }^{1}$ \\ Joe Anthony Fawke, ${ }^{7}$ on behalf of the Cord Pilot Trial Collaborative Group
}

\begin{abstract}
${ }^{1}$ Nottingham Clinical Trials Unit, Queen's Medical Centre, University of Nottingham, Nottingham, UK

${ }^{2}$ Early Life Research Group, Queen's Medical Centre, University of Nottingham, Nottingham, UK ${ }^{3}$ Department of Dermatology, Guy's Hospital, London, UK ${ }^{4}$ Centre for Reviews and Dissemination, Hull York Medical School, University of York, Heslington, York, UK ${ }^{5}$ Neonatal Unit, Liverpool Women's Hospital, Liverpool, UK ${ }^{6}$ Neonatal Intensive Care Unit, City Hospital, Nottingham, UK ${ }^{7}$ Neonatal Unit, Leicester Royal Infirmary, Leicester, UK
\end{abstract}

\section{Correspondence to} Professor Lelia Duley, Nottingham Clinical Trials Unit, Queen's Medical Centre, University of Nottingham, Nottingham NG7 2UH, UK; lelia. Duley@nottingham.ac.uk

Received 21 December 2016 Revised 19 June 2017 Accepted 23 June 2017 Published Online First 18 September 2017

\section{SLinked}

- http://dx.doi.org/10.1136/ archdischild-2017-313416

\section{CrossMark}

To cite: Duley L, Dorling J, Pushpa-Rajah A, et al. Arch Dis Child Fetal Neonatal Ed 2018;103:F6-F14.

\begin{abstract}
Objectives For very preterm births, to compare alternative policies for umbilical cord clamping and immediate neonatal care.
\end{abstract}

Design Parallel group randomised (1:1) trial, using sealed opaque numbered envelopes.

Setting Eight UK tertiary maternity units.

Participants 261 women expected to have a live birth before 32 weeks, and their 276 babies.

Interventions Cord clamping after at least 2 min and immediate neonatal care with cord intact, or clamping within $20 \mathrm{~s}$ and immediate neonatal care after clamping. Main outcome measures Intraventricular haemorrhage (IVH), death before discharge.

Results 132 women (137 babies) were allocated clamping $\geq 2$ min and neonatal care cord intact, and 129 (139) clamping $\leq 20$ s and neonatal care after clamping; six mother-infant dyads were excluded ( 2 , 4) as birth was after $35^{+6}$ weeks, one withdrew (death data only available) $(0,1)$. Median gestation was 28.9 weeks for those allocated clamping $\geq 2$ min, and 29.2 for those allocated clamping $\leq 20 \mathrm{~s}$. Median time to clamping was 120 and $11 \mathrm{~s}$, respectively. 7 of 135 infants (5.2\%) allocated clamping $\geq 2$ min died and 15 of 135 $(11.1 \%)$ allocated clamping $\leq 20$ s; risk difference (RD) $-5.9 \%(95 \% \mathrm{Cl}-12.4 \%$ to $0.6 \%)$. Of live births, 43 of $134(32 \%)$ had IVH vs 47 of $132(36 \%)$, respectively; RD $-3.5 \%$ (-14.9\% to $7.8 \%)$. There were no clear differences in other outcomes for infants or mothers. Conclusions This is promising evidence that clamping after at least 2 min and immediate neonatal care with cord intact at very preterm birth may improve outcome; a large trial is urgently needed.

Trial registration ISRCTN 21456601.

\section{INTRODUCTION}

At birth, if the umbilical cord is not clamped blood flow between baby and placenta may continue for several minutes. ${ }^{1-3}$ This umbilical flow is part of the physiological transition from the fetal to the neonatal circulation; clamping the cord too soon may restrict the infant's ability to cope with this transition. ${ }^{24-8}$ A short delay in cord clamping may increase neonatal blood volume, a longer delay of several minutes may have more substantive advantages, such as smoother cardiorespiratory transition and more stable blood pressure, which for very preterm infants ( $<32$ weeks gestation) may reduce the risk of intraventricular haemorrhage. ${ }^{10}$ Concerns about deferring (delaying) cord clamping include exacerbating jaundice,

\section{What is already known on this topic?}

If the umbilical cord is not clamped flow continues for longer than previously thought, and cord pulsation does not correlate with umbilical flow.

- A short delay in cord clamping may reduce the risk of intraventricular haemorrhage and improve outcome in preterm babies.

- Previous small trials excluded babies requiring resuscitation at birth.

\section{What this study adds?}

- Neonatal stabilisation and resuscitation can be provided with the cord intact.

- Giving neonatal care with the cord intact may improve outcome for the infants born very preterm.

- The effect of resuscitating preterm babies with an intact cord needs to be addressed in a large multicentre trial.

polycythaemia, delayed respiratory support and hypothermia.

The relevant Cochrane review suggests that for infants born preterm deferring cord clamping may improve outcomes at hospital discharge. ${ }^{11}$ However, the 15 trials included were small and have a high risk of bias. None were prospectively registered, and they reported different outcomes, so the possibility of selective outcome reporting is high. Also, data on long-term safety are sparse. Importantly, these studies excluded infants requiring resuscitation at birth, and for very preterm births most trials deferred clamping for $<60$ s. Guidelines for very preterm birth make various recommendations about when to clamp the cord, ${ }^{12-14}$ and advise that clamping should not be delayed if neonatal resuscitation is required. ${ }^{14} 15$ Evaluating timing of cord clamping at very preterm birth is a research priority, particularly for infants requiring resuscitation at birth. $^{15-17}$

Our hypothesis is that for very preterm births a policy of cord clamping after at least $2 \mathrm{~min}$ and providing immediate neonatal care with cord intact, rather than usual care of clamping within $20 \mathrm{~s}$ and neonatal care after clamping, improves outcome for the infants without adverse effects for 


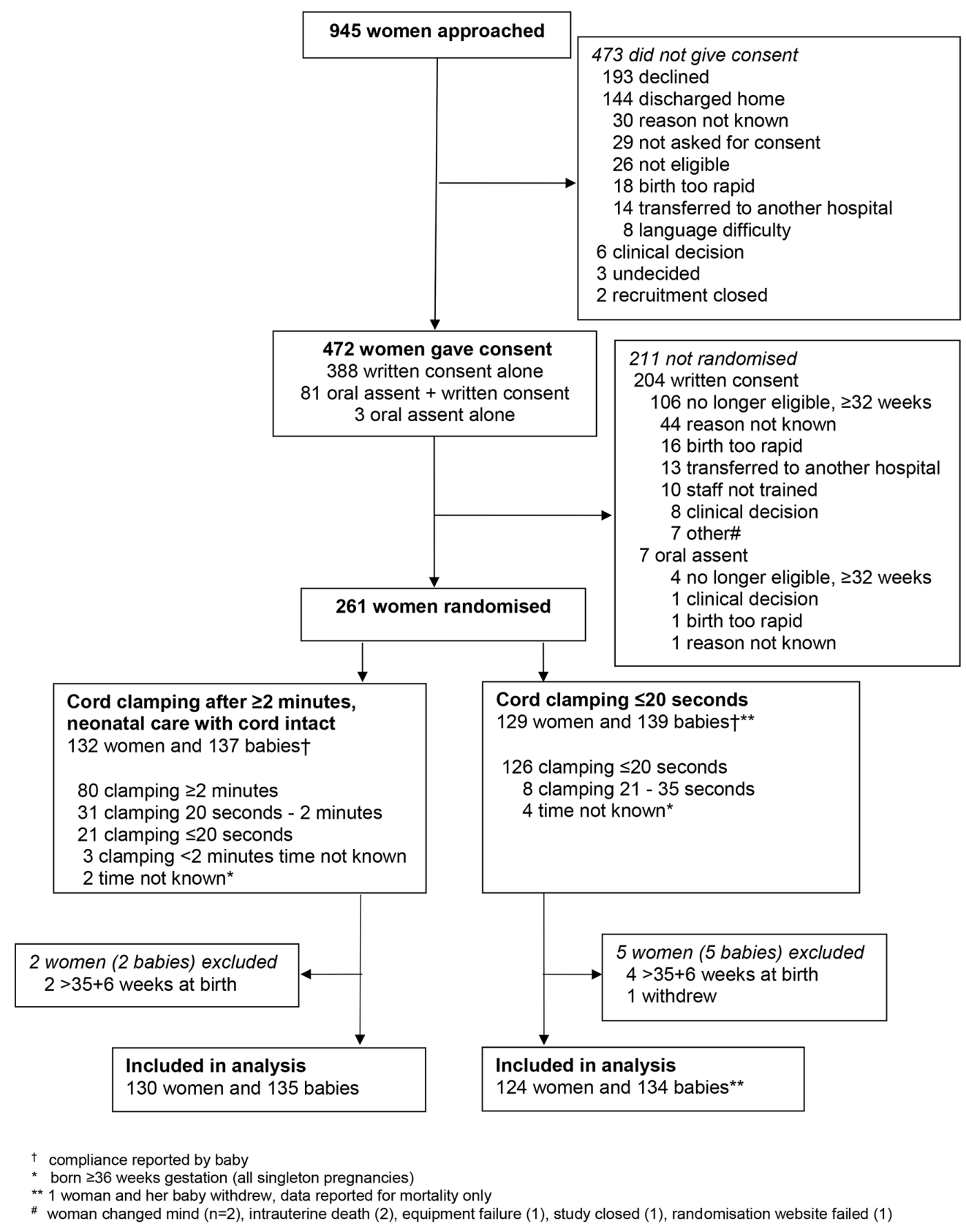

Figure 1 Participant flow.

the women. Having developed strategies for providing stabilisation and resuscitation with the cord intact, ${ }^{18} 19$ we conducted a randomised trial.

\section{METHODS}

Trial design and changes since trial registration

This pragmatic randomised parallel group trial was conducted at eight UK tertiary maternity units with a neonatal intensive care unit. The protocol is published ${ }^{20}$ along with an update, ${ }^{21}$ and these are summarised here. Initially, the study aimed to assess the feasibility of a large multicentre trial and planned to recruit for 1 year. Feasibility was demonstrated and, on the advice of the independent Trial Steering Committee, recruitment continued while funding was sought for the main trial. Recruitment closed in February 2015 when the funding application was unsuccessful.
The sponsor is Nottingham University Hospitals NHS Trust. Trial coordination was at the Nottingham Clinical Trials Unit (NCTU).

As the original protocol was for a pilot trial, outcomes were measures of feasibility and analysis by allocated group was not planned. The statistical analysis plan (SAP) for the extended study was agreed before data were unblinded. For the planned main trial, main outcomes were death before discharge and intraventricular haemorrhage (all grades), hence these were the main outcomes in this SAP. Data presented here are for outcomes at discharge. Follow-up for women at 1 year, and for children at age 2 years (corrected for gestation) will be reported separately.

\section{Parent and parent representative involvement}

Three parent representatives were coinvestigators, involved in identifying the research question, securing funding and designing 
and conducting the study. They advised on plans for recruitment; contributed to developing the oral assent pathway; advised on study implementation and conduct; participated in investigator meetings, including training in explaining the study to women and offering consent and contributed to interpretation and writing up of results. Two parents were independent members of the Trial Steering Committee. Participants in the trial have been thanked for participation, asked for their views of participation and updated on progress. When results are published they will be summarised in lay language.

\section{Participants}

Women were eligible if they were expected to have a live birth before 32 weeks gestation, regardless of mode of birth or fetal presentation. Exclusion criteria were monochorionic twins; triplets or higher-order multiple pregnancy and known major congenital malformation.

\section{Interventions}

Umbilical cord clamping after at least $2 \mathrm{~min}$ and, if needed, immediate neonatal stabilisation and resuscitation with cord intact was compared with clamping within $20 \mathrm{~s}$ and, if needed, immediate neonatal stabilisation and resuscitation after clamping. Waiting at least $2 \mathrm{~min}$ before clamping was based on a balance between waiting until umbilical flow ceased (which may take 3-5 min, or longer), ${ }^{23822}$ and what was acceptable to clinicians. For the intervention group, with cord intact babies were placed onto a firm surface with easy access to resuscitation equipment; either the usual equipment ${ }^{19}$ or a smaller trolley designed for this purpose ${ }^{23}$ moved alongside the mother's bed. For caesarean births, the neonatal resuscitation equipment was covered with sterile drapes, and the neonatologist scrubbed and gowned. For both groups, after cord clamping neonatal care was either beside the mother or at the usual location (side of the room or separate room), at the discretion of the local clinicians. Six sites used their usual resuscitation equipment (153 women recruited) and two the trolley (108). Until cord clamping, the baby was kept at the level of placenta (introitus or mothers' abdomen, or if a caesarean birth the anterior thigh). Clamping within $20 \mathrm{~s}$ with stabilisation and resuscitation after cord clamping was based on the current UK practice, ${ }^{24}$ and previous trials. ${ }^{11}$

For both groups, all other aspects of care including intubation and respiratory support were at the discretion of the attending clinicians. Neonatal care was based on local unit policy and consistent with Resuscitation Council (UK) newborn life support guidelines. ${ }^{14} 25$ Standard equipment was used according to local practice, including plastic sheets or bags, towels and hats, warming mattresses or overhead heaters and saturation monitors.

\section{Outcome measures}

Main outcomes were death before hospital discharge and intraventricular haemorrhage (all grades using the Papile classifica$\operatorname{tion}^{26}$ ). A single assessor reviewed the cranial ultrasound scan reports for intraventricular haemorrhage, blind to the allocated group. Then eight trained clinicians (neonatologists or radiologists) independently adjudicated each scan, blind to allocation. ${ }^{27}$ If the adjudication disagreed with the scan report review, a second independent adjudicator assessed the scan images. Remaining discrepancies were resolved by discussion.

Other outcomes for the baby were: severe intraventricular haemorrhage, ${ }^{26}$ periventricular leukomalacia, blood transfusion, hypothermia $\left(<36^{\circ} \mathrm{C},<35^{\circ} \mathrm{C}\right)$, chronic lung disease (supplemental oxygen or ventilator support at 36 weeks postmenstrual age), ventilation, necrotising enterocolitis (grade 2 or higher), clinical sepsis, treatment for jaundice, treatment for patent ductus arteriosus, treatment for retinopathy of prematurity and duration of hospital stay.

Outcomes for women were: postpartum haemorrhage $(\geq 500$ or $\geq 1000 \mathrm{~mL}$ ), postpartum infection, breast feeding and for vaginal births manual removal of placenta and third stage of labour longer than $30 \mathrm{~min}$. Data were collected up to discharge by research staff at site.

\section{Recruitment and randomisation}

Study information was available in antenatal clinics and wards. Women at risk of very preterm birth were offered participation, and if they accepted gave written consent. Eligibility and consent were checked before randomisation, which was during labour or before caesarean section. If birth was imminent and the attending clinician considered it appropriate, women were offered a brief explanation of the study and offered participation (oral assent). Those who gave oral assent were then randomised. After the birth, these women had an opportunity to discuss the study and were invited to give written consent for participation in follow-up.

Randomisation was by attending clinicians, who took the next sealed consecutively numbered opaque envelope from a ringbinder folder. Sequence generation (1:1) was by computer, stratified by centre with balanced blocks of randomly varying size, created by NCTU. On the envelope was a label to record the date, time, woman's initials, her date of birth and gestation. Once this label was completed she was randomised, even if the envelope was not opened. Inside the envelope was a yellow card instructing when to clamp the cord, and a 'Birth Record' (plus a second for twins) to record information about the third stage of labour and neonatal care at birth. This was completed by clinical staff, and filed in the baby's medical notes. Used envelopes and yellow cards were placed in a locked mailbox, which was emptied regularly. Details from each envelope were entered into the online randomisation log maintained by NCTU. If an envelope was taken but not used, it was returned unopened to NCTU.

During the last few months of recruitment, a secure web-based randomisation system, using the same randomisation sequence, was introduced in three sites to assess its feasibility.

\section{Sample size}

Based on a total of 43600 live births per year at the eight maternity units, we expected $610(1.4 \%)^{28}$ live births to be before 32 weeks; as target accrual was $16 \%-18 \%$ of eligible births we anticipated 100-110 women randomised per year. This was planned as a pilot trial so there was no formal power calculation.

\section{Statistical analysis}

All analyses are based on the groups as randomly allocated (intention to treat). For twin pregnancies, outcome is reported for both babies. Where appropriate, results are presented as relative risk (RR) or risk difference (RD) with 95\% CIs. Women who were randomised but gave birth after $35^{+6}$ weeks are excluded, as outcomes for these babies are different from those born very preterm. For secondary outcomes, missing data for individual items are only reported if they exceeded $1 \%$ of total data available. No formal interim analysis was planned. Data were monitored in 
confidence by an independent Data Monitoring Committee, which met four times.

\section{RESULTS}

Of 945 women approached, 472 (50\%) gave consent (figure 1). Oral assent was offered to 93 women, of whom $84(90 \%)$ assented and of these 77 were randomised. For eight of the 77 women randomised following oral assent birth was not imminent as anticipated, and written consent was given before birth, hence 69 were randomised with oral assent only (33 cord clamping $\geq 2 \mathrm{~min}, 36$ clamping $\leq 20 \mathrm{~s}$ ). For 66 of these women written consent was given after the birth; written consent was not obtained for three, for two their baby died and they did not return for their counselling appointment at which written consent would have been sought and one was transferred to another unit.

Recruitment was from March 2013 to February 2015, and 261 women were randomised (132 cord clamping after at least $2 \mathrm{~min}, 129$ within $20 \mathrm{~s}$ ). Six women were excluded as they gave birth after $35^{+6}$ weeks and one withdrew (outcome data reported only for death before discharge), leaving 254 women for analysis (figure 1). In 2 of the 17 twin pregnancies, one fetus died in utero before randomisation, so 269 babies are included in analysis. Time between randomisation and birth was within 30 min for 81 women (32\%), within 1 hour for $128(50 \%)$ and within 2 hours for 178 (70\%); 20 (8\%) gave birth 24 hours or more after randomisation.

\section{Baseline characteristics}

The groups were balanced at trial entry (table 1). A third of women were randomised before 28 weeks, and two-thirds before 30 weeks. One hundred forty-four (57\%) were in their first pregnancy and just over half had a caesarean birth. Of the babies, 143 (53\%) were males (71/135 clamping after $\geq 2 \mathrm{~min}$ vs $72 / 134$ clamping $\leq 20$ s).

\section{Compliance with the allocated intervention}

Median time to cord clamping was 120 s (IQR 36-134), for those allocated clamping after at least $2 \mathrm{~min}$ and neonatal care with cord intact, and $11 \mathrm{~s}$ for those allocated clamping within $20 \mathrm{~s}$ and neonatal care after clamping (IQR 10-20). In the intervention group, cord clamping was after at least 2 min for 80 (59\%) babies and after $20 \mathrm{~s}$ for 111 (82\%) (figure 2). Baseline characteristics were similar for babies with cord clamping after at least $2 \mathrm{~min}$ and those clamped before $2 \mathrm{~min}$; for example, gestation at birth $28^{+0}$ to $31^{+6}$ weeks $64 \%$ vs $63 \%$, respectively; antenatal corticosteroids $87 \%$ vs $94 \%$ and caesarean birth $61 \%$ vs $67 \%$. For the 55 babies for whom clamping was before $2 \mathrm{~min}$, reasons were: cord too short $(21,38 \%)$, an issue that improved with experience; clinical decision $(12,22 \%)$; baby born either membranes intact or with the placenta $(8,15 \%)$; placental abruption (5, $9 \%)$; neonatal team not there in time $(4,7 \%)$; staff error $(4,7 \%)$ and cord snapped $(1,2 \%)$. In the control group, cord clamping was within $20 \mathrm{~s}$ for 126 (94\%). There were no clear differences in compliance based on whether the site used usual resuscitation equipment or the trolley (data not shown). Neonatal care was comparable between the two allocated groups (table 2). For liveborn babies, median birth weight was $1108 \mathrm{~g}$ (IQR 880-1360) in the intervention group and $1180 \mathrm{~g}$ (IQR 900-1418 g) in the control group (figure 2).

A prophylactic uterotonic drug was given to 127 women (98\%) allocated clamping after at least $2 \mathrm{~min}$, and $120(97 \%)$ allocated clamping within $20 \mathrm{~s}$. Administration was before cord
Table 1 Baseline characteristics for the women

\begin{tabular}{|c|c|c|}
\hline & $\begin{array}{l}\text { Clamp } \geq 2 \\
\text { min+neonatal } \\
\text { care with cord } \\
\text { intact }\end{array}$ & $\begin{array}{l}\text { Clamp } \leq 20 \\
\text { s+neonatal } \\
\text { care after } \\
\text { clamping }\end{array}$ \\
\hline & $(n=130)$ & $(n=124)$ \\
\hline Gestation at randomisation (weeks) $\geq 32$ & $1(1 \%)$ & $1(1 \%)$ \\
\hline 30 to $31^{+6}$ & $44(34 \%)$ & $46(37 \%)$ \\
\hline 28 to $29^{+6}$ & $38(29 \%)$ & $42(34 \%)$ \\
\hline 26 to $27^{+6}$ & $25(19 \%)$ & $21(17 \%)$ \\
\hline$<26$ & $22(17 \%)$ & $14(11 \%)$ \\
\hline Gestation at birth (weeks) $\geq 32$ & $2(2 \%)$ & $2(2 \%)$ \\
\hline 30 to $31^{+6}$ & $47(36 \%)$ & $44(35 \%)$ \\
\hline 28 to $29^{+6}$ & $35(27 \%)$ & $43(35 \%)$ \\
\hline 26 to $27^{+6}$ & $25(19 \%)$ & $21(17 \%)$ \\
\hline$<26$ & $21(16 \%)$ & $14(11 \%)$ \\
\hline Age (years), mean (SD) & $30.3(6.1)$ & $29.2(6.6)$ \\
\hline Primiparous & $69(53 \%)$ & $75(60 \%)$ \\
\hline Twin pregnancy & ${ }^{*} 7(5 \%)$ & $10(8 \%)$ \\
\hline \multicolumn{3}{|l|}{ Pregnancy complications } \\
\hline Prelabour rupture of membranes & $43(33 \%)$ & $40(32 \%)$ \\
\hline Spontaneous onset of labour & $31(24 \%)$ & $33(27 \%)$ \\
\hline CTG abnormalities/fetal distress & $21(16 \%)$ & $23(19 \%)$ \\
\hline Pre-eclampsia/PIH & $33(25 \%)$ & $24(19 \%)$ \\
\hline Antepartum haemorrhage/placenta previa & $14(11 \%)$ & $18(15 \%)$ \\
\hline Chorioamnionitis & $15(12 \%)$ & $14(11 \%)$ \\
\hline Fetal growth restriction/small for gestational age & $13(10 \%)$ & $13(10 \%)$ \\
\hline Othert & $5(4 \%)$ & $6(5 \%)$ \\
\hline \multicolumn{3}{|l|}{ In last week received } \\
\hline Magnesium sulfate & $67(52 \%)$ & $49(40 \%)$ \\
\hline Not known & $4(3 \%)$ & - \\
\hline Corticosteroids & $117(90 \%)$ & $111(90 \%)$ \\
\hline Caesarean section & $82(63 \%)$ & $67(54 \%)$ \\
\hline Before labour & 69 & 57 \\
\hline During labour & 13 & 10 \\
\hline Vaginal birth & $48(37 \%)$ & $57(46 \%)$ \\
\hline Breech presentation & 9 & 8 \\
\hline
\end{tabular}

* For two, one twin known intrauterine death before randomisation.

†Clamp $\leq 20$ s: abdominal pain ( $n=2)$, severe asthma (1), pyelonephritis (1), antiphosphate lipid syndrome (1) and not known (1). Clamp $\geq 2 \mathrm{~min}$ : renal failure and diabetes (1), previous cervical surgery (1), coagulopathy (1), Crohn's disease (1) and hypotension (1).

CTG, cardiotocograph; PIH, pregnancy-induced hypertension.

clamping for $40(31 \%)$ and $15(12 \%)$, respectively; however, timing was unknown for $47(37 \%)$ and 34 (28\%).

\section{Outcome for babies}

Overall, 7/135 (5.2\%) babies allocated clamping after at least 2 min and neonatal care with cord intact died compared with $15 / 135$ (11.1\%) allocated clamping within $20 \mathrm{~s}$ and neonatal care after clamping (table 3), RR 0.47 (95\% CI 0.20 to 1.11 ); RD $-5.9 \%(-12.4 \%$ to $0.6 \%)$. The only death of an infant born after 30 weeks gestation was due to congenital anomaly that was not known before randomisation (if it had been the woman would not have been eligible). Excluding this death gives RR 0.50 (0.21 to 1.20$)$, and RD $-5.2 \%(-11.5 \%$ to $1.2 \%)$. The three stillbirths were born before 28 weeks, and were resuscitated at birth; cause of death was intrapartum asphyxia for two, and antepartum infection for one. 


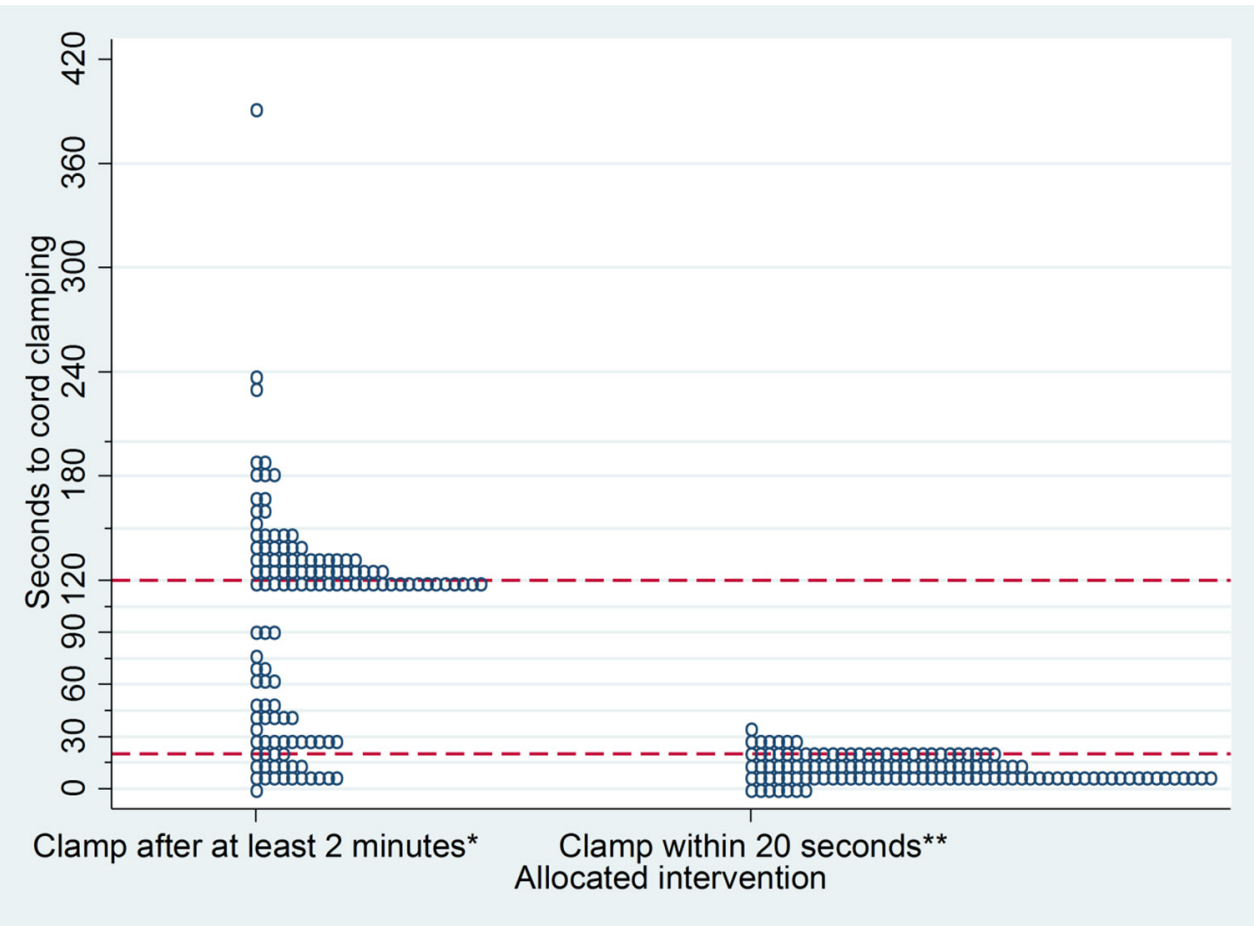

Figure 2 Actual timing of cord clamping for each baby in the two allocated groups. * $\mathrm{n}=135$ babies, seconds to cord clamping not known for 9. ${ }^{* *} n=134$ babies, seconds to cord clamping not known for 9 .

Of live births allocated to the intervention, 43/134 (32\%) had an IVH versus $47 / 132(36 \%)$ allocated to the control group (RR $0.9095 \% \mathrm{CI} 0.64$ to 1.26 ; RD $-3.5 \%$ (95\% CI $-14.9 \%$ to $7.8 \%)$ ) (table 4). Overall, 251 babies (94\%) had a cranial ultrasound scan (127 vs 124, respectively), and both adjudicated scan and review of the original clinical scan report were available for 224/266 (84\%). Diagnosis of IVH was based on adjudication for 81 infants, scan report only for 8 and site data only for 1 . There were no clear differences between the allocated groups for any other outcome (table 4).

\section{Outcome for women}

There was no clear difference between the groups in blood loss at birth $(\geq 500 \mathrm{~mL} 58 / 130$ vs $59 / 124$, RR $0.97,0.71$ to
$1.35 ; \geq 1000 \mathrm{~mL} 11 / 130$ vs $13 / 124 ;$ RR $0.81,0.38$ to 1.73 ), or in any other outcomes for the women (table 5).

\section{DISCUSSION}

This trial suggests that cord clamping after at least 2 min and providing neonatal care with cord intact may improve outcome at discharge for the infants compared with early clamping and neonatal care after clamping, but the CIs are wide and include harm. We achieved a substantial difference of $109 \mathrm{~s}$ between the two groups in median time to clamping, and neonatal care at birth was equivalent in the two groups. For $82 \%$ of infants allocated clamping after at least 2 min umbilical flow continued for longer than in the

Table 2 Neonatal care and newborn life support at birth

\begin{tabular}{|c|c|c|c|c|c|c|}
\hline & \multicolumn{3}{|c|}{ Clamp $\geq 2 \min +$ neonatal care with cord intact $(n=135)$} & \multicolumn{3}{|c|}{ Clamp $\leq 20$ s+neonatal care after clamping $(n=134)$} \\
\hline & Beside mother & $\begin{array}{l}\text { Away from } \\
\text { mother }\end{array}$ & Total & Beside mother & $\begin{array}{l}\text { Away from } \\
\text { mother }\end{array}$ & Total \\
\hline Baby in plastic bag/sheet & $100^{*}$ & 15 & $115(85 \%)$ & $41^{*}$ & 67 & $108(81 \%)$ \\
\hline Mask ventilation & 68 & 32 & $100(74 \%)$ & 14 & 89 & $105+(78 \%)$ \\
\hline Supplemental oxygen & 46 & 38 & $84(62 \%)$ & 9 & 79 & $89 \ddagger(66 \%)$ \\
\hline Airway suction & 39 & 36 & $75(56 \%)$ & 10 & 76 & $87 \ddagger(65 \%)$ \\
\hline Successful intubation & 38 & 40 & $78(58 \%)$ & 10 & 77 & $87(65 \%)$ \\
\hline Surfactant & 28 & 39 & $67(50 \%)$ & 9 & 66 & $75(56 \%)$ \\
\hline Attempted, unsuccessful intubation & 20 & 15 & $35(26 \%)$ & 5 & 33 & $38(28 \%)$ \\
\hline Continuous positive airway pressure & 27 & 17 & $44(33 \%)$ & - & 34 & $34(25 \%)$ \\
\hline Cardiac massage & 3 & 3 & $6(4 \%)$ & 3 & 7 & $10(7 \%)$ \\
\hline Umbilical venous catheterisation & 1 & 2 & $3(2 \%)$ & - & 6 & $6(4 \%)$ \\
\hline Other & - & $1 \S$ & $1(1 \%)$ & - & - & - \\
\hline
\end{tabular}

* Placed in plastic bag beside mother, received all other care at roomside; $n=14$ clamp $\geq 2$ min, $n=26$ clamp $\leq 20$ s. 
Table 3 Mortality for the baby before discharge from hospital

\begin{tabular}{|c|c|c|}
\hline & $\begin{array}{l}\text { Clamp } \geq 2 \\
\text { min+neonatal care } \\
\text { with cord intact }\end{array}$ & $\begin{array}{l}\text { Clamp } \leq 20 \\
\text { s+neonatal care } \\
\text { after clamping }\end{array}$ \\
\hline & $(n=135)$ & $(n=135)^{*}$ \\
\hline Death & $7(5 \%)$ & $15(11 \%)$ \\
\hline Stillbirth & 1 & 2 \\
\hline Early neonatal death & 3 & 7 \\
\hline Late neonatal death & 2 & 5 \\
\hline Postneonatal death & 1 & 1 \\
\hline \multicolumn{3}{|l|}{ Gestation at birth (weeks) } \\
\hline $30-31^{+6}$ & - & 1 \\
\hline $28-29^{+6}$ & 1 & 3 \\
\hline $26-27^{+6}$ & - & 4 \\
\hline$<26$ & 6 & 7 \\
\hline \multicolumn{3}{|l|}{ Cause of death: } \\
\hline Congenital anomaly ${ }^{\dagger}$ & - & 1 \\
\hline Severe pulmonary immaturity & 3 & 3 \\
\hline Intrapartum asphyxia & 1 & 1 \\
\hline IVH & 1 & 2 \\
\hline \multicolumn{3}{|l|}{ Infection } \\
\hline Antepartum & - & 1 \\
\hline Early onset & 1 & 1 \\
\hline Late onset & - & 2 \\
\hline Necrotising enterocolitis & - & 2 \\
\hline Other‡ & 1 & 2 \\
\hline
\end{tabular}

*Includes the baby of one woman who withdrew.

Clamp $\geq 2$ min: prolonged oligohydramnios $(n=1)$; clamp $\leq 20$ s: myocardial

ischaemia (1), prolonged oligohydramnios (1).

†Not known before randomisation.

control group, as the cord was clamped after 20 s. Providing neonatal care with cord intact required a multidisciplinary team approach, planning and training. For the neonatal teams, training was consistent with newborn life support recommendations, ${ }^{14}{ }^{15}$ and included communication with the woman, her partner and other clinical staff. The decision to intubate was based on local hospital policy; the risk of intubation was similar in the two allocated groups, and consistent with the UK practice. ${ }^{29}$ Our study demonstrates that neonatal care with the cord intact is feasible using a range of procedures, and can be done with existing resuscitation equipment ${ }^{19}$ or with a trolley designed for this purpose. ${ }^{18} 23$ This is a more practical and potentially more widely generalisable strategy for supporting continued umbilical flow at birth than in other trials to date.

This study was not powered to detect differences between the allocated interventions. Nevertheless, the most striking result is the difference in death before discharge, this is based on a small number of events however with a wide CI that cannot rule out any difference. Also, there are no clear differences between the groups in IVH or any other serious morbidity that would potentially explain a difference in mortality. Hence, this difference probably reflects the play of chance.

Bringing neonatal care to the mother's bedside allows her and her partner to share the first moments of their child's life. Family presence during resuscitation is standard for other areas of healthcare, where it is preferred by families and appears to be beneficial. ${ }^{30-33}$ Our preliminary work suggests neonatal care beside the mother is acceptable to women and their partners, ${ }^{34}$ and to clinicians. ${ }^{18}{ }^{35}$ Nevertheless, some women reported
Table 4 Neonatal morbidity for liveborn babies

\begin{tabular}{|c|c|c|}
\hline & $\begin{array}{l}\text { Clamp } \geq 2 \\
\text { min+neonatal } \\
\text { care with cord } \\
\text { intact }\end{array}$ & $\begin{array}{l}\text { Clamp } \leq 20 \\
\text { s+neonatal care } \\
\text { after clamping }\end{array}$ \\
\hline & $(n=134)$ & $(n=132)$ \\
\hline Any IVH (grade1-4) & $43(32 \%)$ & $47(36 \%)$ \\
\hline Alive at discharge from hospital & 38 & 42 \\
\hline Severe IVH (grade 3 or 4 ) & $6(4 \%)$ & $7(5 \%)$ \\
\hline Periventricular leukomalacia & $7(5 \%)$ & $8(6 \%)$ \\
\hline Other brain injury & $5(4 \%)$ & $10(8 \%)$ \\
\hline Porencephalic cysts & - & 1 \\
\hline Ventriculomegaly & - & 3 \\
\hline Other* & 5 & 7 \\
\hline Heart rate $<100$ at 1 min & $46+(34 \%)$ & $49(37 \%)$ \\
\hline $\begin{array}{l}\text { Temperature, admission to } \mathrm{NICU}\left({ }^{\circ} \mathrm{C}\right) \text { mean } \\
\text { (SD) }\end{array}$ & $36.7(0.6) \ddagger$ & $36.9(0.8)$ \\
\hline$\leq 36^{\circ} \mathrm{C}$ & $17(13 \%)$ & $14(11 \%)$ \\
\hline$\leq 35^{\circ} \mathrm{C}$ & $2(1 \%)$ & $3(2 \%)$ \\
\hline Blood transfusion (any) & $63(47 \%)$ & $68(52 \%)$ \\
\hline For anaemia & 58 & 66 \\
\hline For hypotension & - & 6 \\
\hline Other indication & 4 & 7 \\
\hline Jaundice requiring treatment & $123(92 \%)$ & $120(91 \%)$ \\
\hline Phototherapy & 123 & 120 \\
\hline Exchange transfusion & - & - \\
\hline Polycythaemia requiring intravenous fluids & - & $1(1 \%)$ \\
\hline Chronic lung disease§ & $40(31 \%)$ & $39(33 \%)$ \\
\hline Ventilation & $100(75 \%)$ & $103(78 \%)$ \\
\hline Duration (days), median (IQR) & $3(1,9)$ & $2(1,9)$ \\
\hline Necrotising enterocolitis & $8(6 \%)$ & $5(4 \%)$ \\
\hline X-ray with perforation or pneumatosis & 7 & 3 \\
\hline Laparotomy & 6 & 3 \\
\hline Suspected necrotising enterocolitis 9 & $15(11 \%)$ & $13(10 \%)$ \\
\hline Sepsis & $72(53 \%)$ & $80(61 \%)$ \\
\hline Positive culture+antibiotics $\geq 5$ days & 30 & 33 \\
\hline Negative culture+antibiotics $\geq 5$ days & 42 & 47 \\
\hline \multicolumn{3}{|l|}{ Treatment for: } \\
\hline Patent ductus arteriosus & $20(15 \%)$ & $20(15 \%)$ \\
\hline Retinopathy of prematurity§ & $5(4 \%)$ & $5(4 \%)$ \\
\hline $\begin{array}{l}\text { Duration of hospital stay** (days) median } \\
\text { (IQR) }\end{array}$ & $57(39,85)$ & $57(38,75)$ \\
\hline Mother's breast milk at discharge ${ }^{* *}+\dagger$ & $71(55 \%)$ & $68(57 \%)$ \\
\hline
\end{tabular}

${ }^{*}$ Clamp $\geq 2$ min: prominent subarachnoid spaces suggestive of atropy $(n=2)$, periventricular cyst (1), absent cavum septum pellucidum (1), occipital cyst (1). Clamp $\leq 20$ s: prominent subarachnoid spaces suggestive of atropy $(n=2)$, periventricular echodensities (1), increased echogenicity of deep white matter (1), mega cysterna (1), ventriculitis (1) and marked ventricular asymmetry (1). †Not known $\mathrm{n}=2$.

‡Temperature not recorded for two babies.

§Babies who survived to 36 weeks postmenstrual age: $n=129$ clamping $\geq 2$ min, $\mathrm{n}=120$ clamping $\leq 20 \mathrm{~s}$. Data collected at 36 weeks postmenstrual age, or discharge whichever happened first.

१Defined as bowel rest+antibiotics $\geq 5$ days.

**Admitted to neonatal unit and alive at discharge: $n=128$ clamping $\geq 2 \mathrm{~min}$; $\mathrm{n}=120$ clamping $\leq 20 \mathrm{~s}$.

††Not known $n=3$ clamping $\geq 2 \min ; n=1$ clamping $\leq 20$ s.

negative emotions, ${ }^{34}$ and clinicians, particularly those with less experience, were concerned about 'performing' in front of the parents. ${ }^{35}$ Few babies in these studies required resuscitation, however, and further evaluation is required. 


\begin{tabular}{|c|c|c|}
\hline & $\begin{array}{l}\text { Clamp } \geq 2 \\
\text { min+neonatal } \\
\text { care with cord } \\
\text { intact }\end{array}$ & $\begin{array}{l}\text { Clamp } \\
\leq 20 \text { s+neonatal } \\
\text { care after } \\
\text { clamping }\end{array}$ \\
\hline & $n=130$ & $n=124$ \\
\hline \multicolumn{3}{|l|}{ Blood loss at birth } \\
\hline$\geq 500 \mathrm{~mL}$ & $58(45 \%)$ & $59(48 \%)$ \\
\hline$\geq 1000 \mathrm{~mL}$ & $11(8 \%)$ & $13(10 \%)$ \\
\hline \multicolumn{3}{|l|}{ For vaginal births* } \\
\hline manual removal of placenta & $5(10 \%)$ & $6(11 \%)$ \\
\hline Third stage $>30 \mathrm{~min}$ & $4(8 \%)$ & $6(11 \%)$ \\
\hline Blood transfusion & $5(4 \%)$ & $3(2 \%)$ \\
\hline Postpartum infection+parenteral antibiotics & $34(26 \%)$ & $29(23 \%)$ \\
\hline Fever $>38^{\circ} \mathrm{C}$ & $8(6 \%)$ & $5(4 \%)$ \\
\hline Duration of hospital stay (days) median (IQR) & $4(2,6)$ & $4(2,6)$ \\
\hline Expressing/breast feeding at discharget & $121(96 \%)$ & $111(93 \%)$ \\
\hline
\end{tabular}

${ }^{*} \mathrm{n}=48$ clamping $\geq 2 \mathrm{~min} ; \mathrm{n}=57$ clamping $\leq 20 \mathrm{~s}$.

tFor women whose babies were alive at time of their discharge, $n=126$

clamping $\geq 2$ min; $n=120$ clamping $\leq 20$ s.

In our study, median birth weight was lower, rather than higher, for infants allocated deferred clamping; suggesting that net change in neonatal blood volume may not be relevant for very preterm births and supporting our hypothesis that continued umbilical flow has a role in the expanding pulmonary circulation during transition to the neonatal circulation.

\section{Strengths and limitations of this study}

Previous trials have largely excluded infants needing immediate resuscitation at birth. ${ }^{15}$ We developed two strategies that enabled us to recruit these high-risk births. First, providing resuscitation with cord intact as needed allowed infants requiring immediate resuscitation at birth to be included. Second, if birth was imminent leaving insufficient time for the usual consent process, women were offered the opportunity to participate through a two-stage oral assent pathway; a quarter of recruitment used this pathway. The two-stage approach seems acceptable to women and to clinicians. ${ }^{36}$ Other strengths are that the study was multicentre and conducted within existing clinical services, hence widely generalisable to similar settings, and that independent adjudication of cranial ultrasound scans improved reliability in ascertainment of IVH. ${ }^{37}$

Although the largest preterm cord clamping trial published to date, this trial was not powered to demonstrate clinically important differences in outcome between the two policies, therefore the key limitation is sample size.

\section{Comparison with other studies}

One previous trial comparing alternative policies for timing of cord clamping (46 babies) has described providing neonatal care with the cord intact, ${ }^{38}$ but the only outcome reported was neonatal blood volume. Other trials comparing alternative policies for cord clamping do not report providing immediate neonatal care with cord intact. One recent trial (150 babies) has evaluated ventilation with cord intact for infants born before 32 weeks gestation, but in this study cord clamping was at $60 \mathrm{~s}$ in both intervention arms. ${ }^{39}$

The Cochrane review includes 15 trials (738 babies) before 37 weeks gestation. ${ }^{11}$ Restricting this to trials largely recruiting before 32 weeks and excluding those evaluating cord milking leaves 12 trials (552 babies) with deferred clamping between 30 and $120 \mathrm{~s}$. In these trials, 8/236 (3.4\%) babies allocated deferred clamping died compared with 14/250 (5.6\%) allocated immediate clamping, and IVH was 31/195 (16\%) vs 50/199 (25\%), respectively. These event rates are substantially lower than in our trial, as high-risk infants requiring resuscitation at birth were not recruited. In our study, the overall mortality at discharge from hospital $(8.1 \%)$ is comparable to that reported for infants of a similar gestation admitted to the 37 UK neonatal units participating in the Vermont Oxford Network (for 1116 inborn infants born between 24 and 31 weeks gestation during 2015, mortality at discharge was $10.8 \%){ }^{40}$ This provides reassurance that we successfully recruited a generalisable group of babies. Diagnosis of any IVH (34\%) is higher than reported in the UK for similar babies $(27 \%),{ }^{40}$ but reassuringly severe IVH is similar $(5 \%$ and $6 \%$, respectively). Hence, the difference in any IVH diagnosis is likely to be due to variation in the grade 1 and 2 IVH, with another potential factor being differences between hospitals in the proportions of infants born before 32 weeks gestation who have a cranial ultrasound scan. Although some previous studies reported that cranial ultrasound scans were conducted blind to the allocation, none have reported independent adjudication of the scan diagnosis.

Other issues with the trials included in the Cochrane Review are that only two appear to have been registered. This may be relevant since most studies reported mortality, the definition (eg, including or excluding major congenital abnormality and/or stillbirth) varied, and of 11 other outcomes none were reported by more than 6 out of the 15 trials: therefore, selective outcome reporting must be at least a possibility. The apparently high compliance noted in some previous trials seems rather implausible and raises the possibility of unreported postrandomisation exclusions, and hence further potential for bias.

Resuscitation guidelines state 'there is insufficient evidence to recommend an appropriate time for clamping the cord in infants who are severely compromised at birth, and that for infants requiring resuscitation, resuscitative intervention remains the immediate priority'. ${ }^{15}$ Clearly, uncertainty remains about when to clamp the cord for infants requiring resuscitative interventions. For preterm infants who are stable at birth and do not require resuscitation, recommendations are to wait at least $30 \mathrm{~s}$ and no longer than 3 min. ${ }^{15} 41$

Cord milking is advocated to increase neonatal blood volume without needing to defer cord clamping. ${ }^{42}$ This technique disrupts umbilical blood flow, however, over-riding autoregulation of blood volume and blood pressure. As a different intervention to cord clamping, with greater potential to do harm, it requires rigorous evaluation. ${ }^{43-45}$

\section{CONCLUSIONS}

A large multicentre trial is urgently needed to confirm whether a long delay ( $2 \mathrm{~min}$ or more) in cord clamping combined with stabilisation and/or resuscitation with cord intact, rather than standard care, really does improve outcome for very preterm births. This study demonstrates that such a trial is feasible.

Acknowledgements The authors thank all the women who participated in this trial, and their families, and to the clinical and research staff at the sites. The authors also thank Diane Whitham and Gill Bumphrey for preparation of the randomisation envelopes; to Alec Whitham for making the mail boxes and to Dushyant Batra, Louise Hattingh, Shoaib Khan and Kiran Yajamanyam for their assistance with the cranial ultrasound scan adjudication.

Collaborators Cord Pilot Trial Collaborative Group: Nottingham Clinical Trials Unit: Lelia Duley (chief investigator). Lindsay Armstrong-Buisseret (trial manager from November 2015). Brian Barnes (data coordinator). Lucy Bradshaw (statistician). Natalie Hutchings (trial administrator). Eleanor Mitchell (senior trial manager). 
Angela Pushpa-Rajah (trial manager May 2013-July 2015). Keith Whittaker (database programmer). Trial Management group: Lucy Bradshaw, Jon Dorling, Lelia Duley, Eleanor Mitchell, Angela Pushpa-Rajah (chair). Trial Steering Committee: Lucy Bradshaw (statistician), Kate Branchett, Mike Clarke (chair), Richard Cooke, Jon Dorling (neonatologist), Lelia Duley (chief investigator), Liz Goddard, Sara Kenyon, Angela Pushpa-Rajah (trial manager), Philip Steer. Data Monitoring Committee: Douglas Altman (chair), Declan Devane, Andrew Shennan, Ben Stenson. Patient and public involvement: Jane Abbott (Bliss, the special care baby unit charity) (until June 2014), Zoe Chivers (Bliss), Gill Gyte (NCT, National Childbirth Trust). Cranial ultrasound adjudication: Lindsay Armstrong-Buisseret, Lucy Bradshaw, Robert Dineen, Jon Dorling, Lelia Duley, Eleanor Mitchell. Clinical advisors: David Field, Jim Thornton, William Tarnow-Mordi. Writing committee: Lucy Bradshaw, Rob Dineen, Jon Dorling, Lelia Duley (chair) and Eleanor Mitchell. Collaborators by site (total number of women recruited). Aberdeen Maternity Hospital (13): Medhat Ezzat, Nikolaus Kau, Vijender Kistareddy, Annie Robertson: Bradford Royal Infirmary (43): Amal Mighell, Sam Oddie, Kelly Young City Hospital (39): Dushyant Batra, Bernard Schoonakker, Carys Smith, Kumar Swamy Homerton University Hospital (18): Narendra Aladangady, Amit Kandhari, Elaine van der. Pool: Leicester Royal Infirmary (39): Ros Astles, Joe Fawke, Marie Hubbard, Penny McParland Liverpool Women's Hospital (69): Margaret Thomas, Andrew Weeks, Charles William Yoxall New Cross Hospital (14): Kate Cheshire, David Churchill, Tilly Pilley, Melanie Sutcliffe Queen's Medical Centre (26): George Bugg, Jon Dorling, Yvonne Hooton, Gill Kirkwood.

Contributors The study was conceived by LD and GG. The protocol, trial procedures and strategies for recruitment and consent were developed in consultation with a board range of stakeholders including parent representatives, clinicians, researchers, the NIHR programme grant steering group and the Nottingham Clinical Trials Unit. The paper was drafted by LD, with comments from other authors. All authors have read and agreed the final paper.

Funding This trial is independent research funded by the National Institute for HealthResearch (NIHR) under its Programme Grants for Applied Research funding scheme (RPPG-0609-10107). The views expressed are those of the authors and not necessarily thoseof the NHS, the NIHR or the Department of Health. The funder had no role in study design, conduct, analysis or reporting.

Competing interests All authors declare no support from any organisation for the submitted work other than the NIHR programme grant; no financial relationships with any organisations that might have an interest in the submitted work in the previous three years; the grant funded research included development of a neonatal resuscitation trolley now marketed as 'LifeStart' and purchased by two sites for use in this trial, several authors were involved in development of the trolley but have no further relationship with the manufacturer; no other relationships or activities that could appear to have influenced the submitted work.

\section{Patient consent Obtained.}

Ethics approval Nottingham REC 2 (NRES reference 12/EM/0283).

Provenance and peer review Not commissioned; externally peer reviewed.

Data sharing statement Patient level data and the dataset presented here are available from the corresponding author. Participant consent for data sharing was not obtained but the presented data are anonymised and risk of identification is low.

Open Access This is an Open Access article distributed in accordance with the terms of the Creative Commons Attribution (CC BY 4.0) license, which permits others to distribute, remix, adapt and build upon this work, for commercial use, provided the original work is properly cited. See: http://creativecommons.org/licenses/by/4.0/

(c) Article author(s) (or their employer(s) unless otherwise stated in the text of the article) 2018. All rights reserved. No commercial use is permitted unless otherwise expressly granted.

\section{REFERENCES}

1 Dawes GS. Foetal and neonatal physiology; a comparative study of the changes at birth [by] Geoffrey S Dawes. Chicago: Year Book Medical Publishers, 1968. Chapter 13

2 Boere I, Roest AA, Wallace E, et al. Umbilical blood flow patterns directly after birth before delayed cord clamping. Arch Dis Child Fetal Neonatal Ed 2015;100:F121-5.

3 Farrar D, Airey R, Law GR, et al. Measuring placental transfusion for term births: weighing babies with cord intact. BJOG 2011;118:70-5.

4 Gunther M. The transfer of blood between baby and placenta in the minutes after birth. Lancet 1957:272:1277-80.

5 Ersdal HL, Linde J, Mduma E, et al. Neonatal outcome following cord clamping after onset of spontaneous respiration. Pediatrics 2014:134:265-72.

6 Committee on Obstetric Practice, American College of Obstetricians and Gynecologists. Committee Opinion No.543: Timing of umbilical cord clamping after birth. Obstet Gynecol 2012;120:1522-6.

7 Bhatt S, Alison BJ, Wallace EM, et al. Delaying cord clamping until ventilation onset improves cardiovascular function at birth in preterm lambs. J Physiol 2013;591:2113-26.
8 Vijayaselvi R, Abraham A, Kumar M, et al. Measuring umbilical flow and placental transfusion for preterm births: weighing babies at 33-36 weeks gestation with cord intact. 1st congress of joint European Neonatal Societies, 2015

9 Hofmeyr GJ, Bolton KD, Bowen DC, et al. Periventricular/intraventricular haemorrhage and umbilical cord clamping. Findings and hypothesis. S Afr Med J 1988;73:104-6.

10 Hofmeyr GJ, Bex PJ, Skapinker R, et al. Hasty clamping of the umbilical cord may initiate neonatal intraventricular hemorrhage. Med Hypotheses 1989;29:5-6.

11 Rabe H, Diaz-Rossello JL, Duley L, et al. Effect of timing of umbilical cord clamping and other strategies to influence placental transfusion at preterm birth on maternal and infant outcomes. Cochrane Database Syst Rev 2012;8:CD003248.

12 RCOG Scientific Advisory Committee. Clamping of the umbilical cord and placental transfusion. London: Royal College of Obstetricians and Gynaecologists, 2015.

13 National Institute for Health and Care Excellence. Preterm labour and birth. London: NICE Guideline, 2015.

14 Richmond S, Wyllie J. European Resuscitation Council Guidelines for Resuscitation 2010 Section 7. Resuscitation of babies at birth. Resuscitation 2010;81:1389-99.

15 Wyllie J, Bruinenberg J, Roehr CC, et al. European Resuscitation Council Guidelines for Resuscitation 2015: Section 7. Resuscitation and support of transition of babies at birth. Resuscitation 2015;95:249-63.

16 Tarnow-Mordi WO, Duley L, Field D, et al. Timing of cord clamping in very preterm infants: more evidence is needed. Am J Obstet Gynecol 2014;211:118-23.

17 Duley L, Uhm S, Oliver S, et al. Top 15 UK research priorities for preterm birth. Lancet 2014:383:2041-2.

18 Thomas MR, Yoxall CW, Weeks AD, et al. Providing newborn resuscitation the mother's bedside: assessing the safety, usability and acceptability of a mobile trolley. BMC Pediatr 2014;14:135

19 Schoonakker B, Dorling J, Oddie S, et al. Bedside resuscitation of preterm infants with cord intact is achievable using standard resuscitaire. Oporto, Portugual: European Society for Pediatric Research, 2013. http://www.espr.info/templates/espr/images/pdf/ 9349_abstract_book_-_25sett13-it-it.pdf.

20 Pushpa-Rajah A, Bradshaw L, Dorling J, et al. Cord pilot trial - immediate versus deferred cord clamping for very preterm birth (before 32 weeks gestation): study protocol for a randomized controlled trial. Trials 2014;15:258.

21 Bradshaw LE, Pushpa-Rajah A, Dorling J, et al. Cord pilot trial: update to randomised trial protocol. Trials 2015;16:407

22 Batey N, Dorling J, Oddie S, et al. Placental transfusion in preterm infants: how long does the transfusion last? Oporto, Portugual: European Society for Pediatric Research, 2013.

23 Weeks AD, Watt $P$, Yoxall CW, et al. Innovation in immediate neonatal care: development of the Bedside Assessment, Stabilisation and Initial Cardiorespiratory Support (BASICS) trolley. BMJ Innov 2015;1:53-8.

24 Farrar D, Tuffnell D, Airey R, et al. Care during the third stage of labour: a postal survey of UK midwives and obstetricians. BMC Pregnancy Childbirth 2010;10:23.

25 Perlman JM, Wyllie J, Kattwinkel J, et al. Part 11: Neonatal resuscitation: 2010 International Consensus on Cardiopulmonary Resuscitation and Emergency Cardiovascular Care Science With Treatment Recommendations. Circulation 2010;122(16 Suppl 2):S516-38.

26 Papile LA, Burstein J, Burstein R, et al. Incidence and evolution of subependymal and intraventricular hemorrhage: a study of infants with birth weights less than 1,500 gm. J Pediatr 1978;92:529-34.

27 Bradshaw LE, Pushpa-Rajah A, Dorling J, et al. Cord pilot trial: update to randomised trial protocol. Trials 2015;16:407.

28 Moser K, Macfarlane A, Chow YH, et al. Introducing new data on gestation-specific infant mortality among babies born in 2005 in England and Wales. Health Stat Q 2007;35:13-27.

29 Singh Y, Oddie S. Marked variation in delivery room management in very preterm infants. Resuscitation 2013;84:1558-61.

30 Critchell CD, Marik PE. Should Family Members Be Present During Cardiopulmonary Resuscitation? A Review of the Literature. American Journal of Hospice and Palliative Medicine 2007:24:311-7.

31 Boie ET, Moore GP, Brummett C, et al. Do parents want to be present during invasive procedures performed on their children in the emergency department? A survey of 400 parents. Ann Emerg Med 1999;34:70-4.

32 Moons P, Norekvål TM. European nursing organizations stand up for family presence during cardiopulmonary resuscitation: a joint position statement. Prog Cardiovasc Nurs 2008;23:136-9.

33 Resuscitation Council. Should relatives witness resuscitation? UK: Resuscitation Council, 1996.

34 Sawyer A, Ayers S, Bertullies S, et al. Providing immediate neonatal care and resuscitation at birth beside the mother: parents' views, a qualitative study. BMJ Open 2015;5:e008495.

35 Yoxall CW, Ayers S, Sawyer A, et al. Providing immediate neonatal care and resuscitation at birth beside the mother: clinicians' views, a qualitative study. $B M$ Open 2015:5:e008494

36 Ayers S, Sawyer A, Chhoa C, et al. Clinicians' and womens' experiences of two consent pathways in a trial of timing of clamping at very preterm birth: a qualitative study. Trials 2015;16:1. 
37 Dorling J, Duley L, Bradshaw L, et al. Impact of independent Adjudication of Neonatal Cranial Ultrasound Scans in a Randomised Trial. Geneva: European Academy of Paediatric Societies, 2016.

38 Aladangady N, McHugh S, Aitchison TC, et al. Infants' blood volume in a controlled trial of placental transfusion at preterm delivery. Pediatrics 2006;117:93-8.

39 Katheria A, Poeltler D, Durham J, et al. Neonatal Resuscitation with an Intact Cord: A Randomized Clinical Trial. J Pediatr 2016;178:75-80.

40 Boyle E, Manktelow B, Field D, et al. The neonatal survey report 2014. The Infant Mortality and Morbidity Studies, 2014. http://www2leacuk/departments/healthsciences/research/timms/reports

41 NICE: Clinical Guidelines. Preterm Labour and Birth. London: NICE, 2015.
42 Hosono S, Mugishima H, Fujita H, et al. Umbilical cord milking reduces the need for red cell transfusions and improves neonatal adaptation in infants born at less than 29 weeks' gestation: a randomised controlled trial. Arch Dis Child Fetal Neonatal Ed 2008;93:F14-19.

43 Nolan JP, Hazinski MF, Aickin R, et al. Part 1: Executive summary. Resuscitation 2015:95:e1-31.

44 Rabe H, Jewison A, Alvarez RF, et al. Milking compared with delayed cord clamping to increase placental transfusion in preterm neonates: a randomized controlled trial. Obstet Gynecol 2011;117(2 Pt 1):205-11.

45 Katheria AC, Truong G, Cousins L, et al. Umbilical Cord Milking Versus Delayed Cord Clamping in Preterm Infants. Pediatrics 2015;136:61-9. 